\title{
Reactive capture using metal looping: the effect of oxygen
}

\author{
George R. M. Dowson, (D) * Joshua Cooper and Peter Styring*
}

Received 4th January 2021, Accepted 16th February 2021

DOI: $10.1039 / \mathrm{d} 1 \mathrm{fd} 00001 \mathrm{~b}$

In the effort to create a sustainable future economy, the ability to directly convert dilute gas-phase $\mathrm{CO}_{2}$ in waste gas streams into useful products would be a valuable tool, which may be achievable using Grignard reagents as both the capture and the conversion materials. The magnesium salt by-product can be recovered, and metallic magnesium regenerated through conventional high-efficiency electrolysis. This stoichiometric approach is known as metal looping, where the magnesium acts as the energy vector for the capture and conversion, allowing both to occur at room temperature and atmospheric pressure. However, the process has only previously been demonstrated with $12 \% \mathrm{CO}_{2}$ in nitrogen mixtures. If we consider this process in a real post-combustion flue gas conversion scenario, the sensitivity of Grignard reagents to other gases (and water vapour) must be considered. While some of these gases and the water vapour are relatively easily removed, in most flue gas streams the most common other gas present, oxygen, would be far more challenging to excise, and oxygen is known to react with Grignard reagents, albeit slowly. In order to determine if higher oxygen concentrations could be tolerated, allowing the possibility of a variety of relatively inexpensive and possibly profitable direct $\mathrm{CO}_{2}$ conversion pathways to be developed, a range of industrially relevant $\mathrm{CO}_{2} / \mathrm{O}_{2}$ mixtures were made and carefully bubbled through phenylmagnesium bromide solutions.

\section{Introduction}

The drive to net-zero proposed by many nations, whereby carbon dioxide $\left(\mathrm{CO}_{2}\right)$ emissions are reduced by a target date, requires a new way of thinking and a suite of mitigation technologies as there is no single, silver bullet. If the famous Lansink hierarchy of waste management (reduce, reuse, recycle, recover, dispose) is applied to interventions in $\mathrm{CO}_{2}$ mitigation, including treating it as a waste, we can see the hierarchy of first avoiding new fossil carbon entering the supply chain, then the recycling and reuse of the $\mathrm{CO}_{2}$ in carbon capture and utilisation (CCU), before finally recovering and disposing of it as in carbon capture and storage 
(CCS). ${ }^{1}$ While complementary parts of this hierarchy, CCS and CCU have different approaches and consequently employ different solutions to their respective challenges. A key challenge in $\mathrm{CCU}$ is driving down the cost of the $\mathrm{CO}_{2}$-containing product so that the price differential with the counterfactual fossil-derived material is low, promoting a transition to the sustainable alternative. Guidelines for the techno-economic analysis of CCU processes and products has been reported and hotspots include hydrogen production for reduction reactions and the carbon capture or purification steps. ${ }^{2}$ The latter is interesting as elimination of any capture and purification steps can dramatically reduce costs. The problem is that typical flue gases contain not only carbon dioxide but nitrogen, which is inert so not a problem; nitrogen and sulfur oxides, which can be stripped out prior to reaction; and oxygen which is a potential problem as it may be more reactive than the $\mathrm{CO}_{2}$ in any $\mathrm{CCU}$ reactions, leading to by-products, potential high energy reactions and catalyst damage.

The key challenges in using carbon dioxide as a carbon-source for a product is its low reactivity and high oxidation state, often requiring reduction before any reaction can proceed. Furthermore, to maximise the emissions impact, the carbon dioxide has to be taken from dilute gas mixture sources, which would otherwise typically be released into the atmosphere, such as flue gases. While this is a highly active area of development in terms of climate solutions incorporating CCS, most benchmark and proposed carbon capture processes have enormous energetic, capital and process costs. For example, the benchmark amine-capture route, using monoethanolamine (MEA) when applied to coal power plants, decreases the plant efficiency by $25-40 \%$ and would increase the cost of electricity produced by the retrofitted plant by over $70-100 \% .^{3,4}$

In simple energy terms, the capture cost for $\mathrm{CO}_{2}$ using the MEA system amounts to approximately 3-4 $\mathrm{MJ}$ per $\mathrm{kg} \mathrm{CO}_{2} .{ }^{5}$ For comparison, and to illustrate the energy costs, reducing $1 \mathrm{~kg}$ of $\mathrm{CO}_{2}$ to $\mathrm{CO}$ directly (generating 0.5 equivalents of oxygen) would require a minimum of $5.84 \mathrm{MJ}$ per $\mathrm{kg} \mathrm{CO}_{2} \cdot{ }^{6}$ Similarly, if hydrogen is to be used as the reducing agent for $\mathrm{CO}_{2}$, the synthesis by water electrolysis of one stoichiometric equivalent of hydrogen to $1 \mathrm{~kg}$ of $\mathrm{CO}_{2}$ (22.72 moles) would, at a high $80 \%$ efficiency, bring the total to $7.14 \mathrm{MJ}$ per $\mathrm{kg} \mathrm{CO}_{2}$ leaving, in principle, humid CO only as the reaction product. This would then require further conversion into a useful product, with additional hydrogen cost. Note also that products from $\mathrm{CO}_{2} / \mathrm{H}_{2}$ or $\mathrm{CO} / \mathrm{H}_{2}$ mixtures also typically require further energy input in the form of high pressure and temperature reaction conditions.

It has been incorrectly stated on numerous occasions that the low reactivity of carbon dioxide means that utilisation processes should not be pursued and that instead amine capture processes should be investigated for use in CCS. However, this approach is contradictory as amine capture is itself a chemical reaction where the $\mathrm{CO}_{2}$ is stripped from the flue gas to produce the carbamate salt which is isolated and then thermally degraded back to the amine and pure $\mathrm{CO}_{2}{ }^{7}$ Therefore, if a chemical reaction could be used that produces a desirable product directly, then the process becomes simplified, intensified and lower cost.

An alternative approach, investigated previously by the authors, used sacrificial Grignard reagents to capture and convert $\mathrm{CO}_{2}$ directly from the gas phase to a range of potentially valuable carboxylic acid products under room temperature conditions. ${ }^{8}$ Since the by-product of the reaction, the magnesium dihalide, is the commercial feedstock for highly efficient magnesium production by electrolysis, 
in principle the sacrificial Grignard reagent could be regenerated. ${ }^{9}$ This approach would combine the capture and conversion of the $\mathrm{CO}_{2}$ into a single exothermic room-temperature process, which would allow the overall conversion of an alcohol into the carboxylic acid of the next member of the homologous series, with a carbon chain one atom longer than the starting alcohol (Fig. 1).

Naturally, the magnesium electrolysis is the indirect driving force for the reaction scheme shown in Fig. 1, by both reducing the carbon dioxide, to allow formation of the new $\mathrm{C}-\mathrm{C}$ bond, and also essentially driving the capture of the $\mathrm{CO}_{2}$ from the gas phase. Each kilogram of $\mathrm{CO}_{2}$ captured and reacted in this fashion will require $0.552 \mathrm{~kg}$ of magnesium, which can be electrolysed from magnesium dihalide with an energy cost of $14-26 \mathrm{MJ}$ per $\mathrm{kg} \mathrm{CO}_{2} \cdot{ }^{10}$ Comparing this energy cost with that calculated previously of a more traditional carbon dioxide utilisation (CDU) route may initially seem unfavourable, however, it should be considered that the Grignard method combines the capture of the $\mathrm{CO}_{2}$ and its conversion into a potentially useful product directly at room temperature and atmospheric pressure.

This production of a useful product directly may be the key benefit of the Grignard route over conventional capture and conversion, which typically focuses on simple bulk chemicals such as formaldehyde and methanol (which requires additional hydrogen). By comparison, the opportunity for the creation of more complex and valuable compounds, allows the value-added by the addition of the $\mathrm{CO}_{2}$ molecule to the starting alcohol to be large enough for easier overall profitability. This is an issue for potential $\mathrm{CO}_{2}$-generated products as they tend be low value, with current prices around US $\$ 200$ per tonne for formaldehyde and US\$400 per tonne for methanol. Although the total global volumes of $\mathrm{CO}_{2}$ that can be used for the manufacture of specific high-value chemicals is significantly lower, their manufacture from, or partially from, $\mathrm{CO}_{2}$ can act as the "thin end of the wedge" for getting CDU products into the marketplace.

One example of a high value $\mathrm{CO}_{2}$-generated product would be the synthesis of terephthalic acid, used for the production of PET (polyethylene terephthalate) using $\mathrm{CO}_{2}$. Here, 1,4-dibromobenzene, which can be selectively yielded from the reaction of benzene and bromine over zeolites, can be converted into di-Grignard

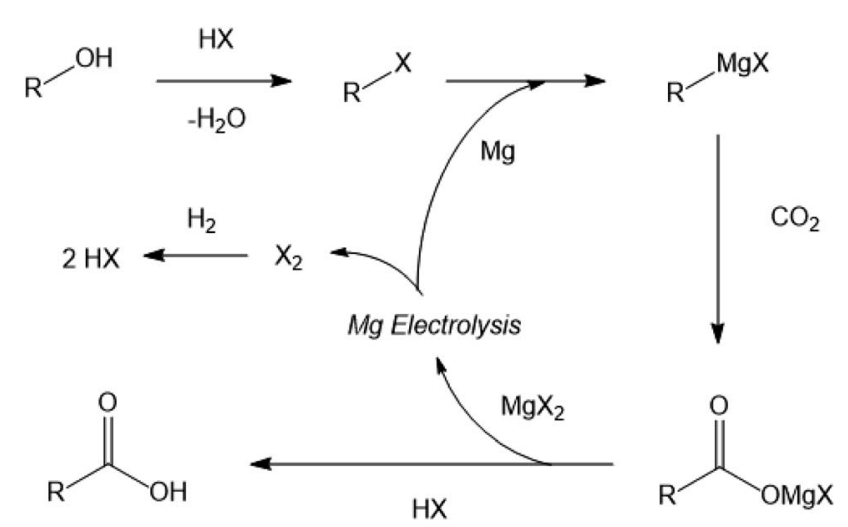

Fig. 1 Generalised scheme for the formation of a higher carboxylic acid from an alcohol and $\mathrm{CO}_{2}$ using regenerable Grignard reagents. 


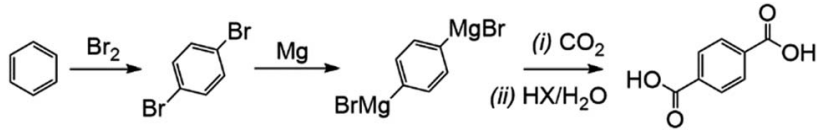

Fig. 2 Synthesis of terephthalic acid from benzene and $\mathrm{CO}_{2}$ via di-Grignard reagent intermediates.

agents. ${ }^{11}$ Exposure to $\mathrm{CO}_{2}$ then allows the terephthalic acid product to form at room temperature without the risk of the formation of discolouring aldehyde byproducts which also interfere with PET polymerisation from the conventional route of para-xylene oxidation. ${ }^{12}$ This is also a relatively rare example of a $\mathrm{CO}_{2}$ utilisation/conversion route that does not require molecular hydrogen to proceed (Fig. 2).

The putative market value added to the benzene by the utilisation of two $\mathrm{CO}_{2}$ molecules in this fashion is approximately US\$1320 per tonne of benzene converted, using average 2017/18 prices, as $1 \mathrm{~kg}$ of benzene reacts, via the Grignard, with $1.13 \mathrm{~kg} \mathrm{CO}$ to yield $2.13 \mathrm{~kg}$ terephthalic acid. ${ }^{13}$

However, there is also a significant potential issue with using Grignard reagents as the basis for a combined capture and conversion route for $\mathrm{CO}_{2}$, which is the presence of other gases and vapour in waste gas streams, particularly oxygen and water, both of which degrade Grignard reagents and are the reason that Grignard chemistry is always performed in an inert atmosphere. ${ }^{14}$ Of the two, water is most damaging to the Grignard reagents, which are well-known to be particularly sensitive to water and decompose readily and violently, as shown in Fig. 3.

The violence and rapid rate of this reaction means that water vapour would certainly have to be removed from the waste gas streams to allow the $\mathrm{CO}_{2}$ to be reacted as shown previously, and this energy cost would be dependent on the water content of the flue gas. However, it is not known whether the oxygen would also have to be removed from the gas stream to prevent the build-up of either hazardous compounds or to avoid the risk of fire, as Grignard reagents are considered to be pyrophoric. ${ }^{15}$ For this reason, relatively few studies have been carried out on the effect of oxygen on Grignard reagents, with the exception of investigations into the synthesis of organic hydroperoxides. ${ }^{16}$ Furthermore, even if the products of the reaction of the Grignard reagent with oxygen do not pose a safety hazard, they could reduce the yield of the desired product and introduce undesirable by-products.

While water can relatively easily be removed from gas streams by desiccants and/or condensation, oxygen removal is far more challenging, and typically relies on combustion to consume the oxygen followed by separation of the water and $\mathrm{CO}_{2}$ by-products. ${ }^{17} \mathrm{~A}$ further factor is that the concentrations of oxygen in industrial flue gases varies widely across different sectors and processes as shown in Table 1.

For example, in Table 1 two figures are given for steel manufacturing as there are multiple emission points across a steel plant, with significant differences in composition. The first of the two steel figures combines the emissions of the lime production and coke oven/production, and the latter is the combined emissions of the steam generators and hot stoves. However, even in the part of steel 


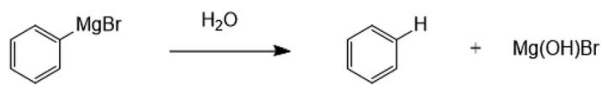

Fig. 3 Degradation of phenylmagnesium bromide with water to produce benzene and hydroxymagnesium bromide.

Table 1 Average dry flue gas compositions for various waste gas sources (in volume percent $)^{18}$

\begin{tabular}{lccl}
\hline $\begin{array}{l}\text { Total\% } \\
\mathrm{CO}_{2}\end{array}$ & $\begin{array}{l}\text { Total\% } \\
\mathrm{O}_{2}\end{array}$ & Percent $\mathrm{O}_{2}$ in binary $\mathrm{CO}_{2} / \mathrm{O}_{2}$ mixture\% & \\
& & \\
12.8 & 7.2 & 36 & Waste gas sources \\
14.0 & 2.9 & 17 & Coal power \\
3.9 & 14 & 78 & Oil power \\
10.8 & 10.9 & 50 & Gas power \\
30.0 & 10.0 & 25 & Waste incineration \\
21.4 & 7.1 & 25 & Cement production \\
29.1 & 0.8 & 2.7 & Steel (low $\mathrm{CO}_{2}$ processes) \\
\multicolumn{7}{l}{ Percentage $\mathrm{O}_{2}$ with respect to $\mathrm{CO}_{2}$ and $\mathrm{O}_{2}$ only, ignoring other gases. Calculated from $\left[\mathrm{O}_{2}\right] /$} \\
{$\left[\mathrm{O}_{2}\right]\left[\mathrm{CO}_{2}\right]$}
\end{tabular}

manufacturing with the lowest oxygen content in its flue gas, the oxygen content is not negligible and may still be sufficient to cause significant undesirable reactivity with Grignard reagents.

To determine whether the presence of oxygen in a hypothetical dry flue gas mixture could still act as a potential source of carbon for Grignard chemistry and thus reactive direct capture of $\mathrm{CO}_{2}$, a series of dry binary $\left(\mathrm{CO}_{2} / \mathrm{O}_{2}\right)$ and ternary $\left(\mathrm{CO}_{2} / \mathrm{O}_{2} / \mathrm{N}_{2}\right)$ gas mixtures were bubbled through a solution of phenylmagnesium halide in THF until the gas supply was exhausted. After quenching, the resulting mixtures were analysed by quantitative ${ }^{1} \mathrm{H}$ NMR to determine the extent of the reaction and the selectivity to the $\mathrm{CO}_{2}$-based product, benzoic acid.

\section{Results and discussion}

\section{Effect of oxygen on the yield of carboxylic acid}

To determine the effect of the presence of oxygen on the yield of the $\mathrm{CO}_{2}$-derived products in a Grignard reaction, phenylmagnesium chloride was chosen due to the relatively low volatility of the aromatic products of both the main benzoic acid reaction (shown in Fig. 4) and any potential by-products including the hydrolysis product, benzene (see Fig. 3).

The main anticipated by-product was phenol, formed via a two-step reaction and subsequent quench as shown in Fig. 5. The initial product of the reaction with oxygen would be the magnesium halide salt of phenyl hydroperoxide (1), which would then disproportionate with another molecule of phenylmagnesium halide to produce two equivalents of the phenoxy magnesium halide (2) before acid quenching to form two molecules of phenol. ${ }^{19}$

Note also that the Grignard- $\mathrm{CO}_{2}$ and both Grignard $-\mathrm{O}_{2}$ reaction routes, which form benzoic acid and phenol respectively, require overall one molecule of 
<smiles>[X][Y9](=O)c1ccccc1</smiles>

Fig. 4 Synthesis of benzoic acid from carbon dioxide and a Grignard reagent.

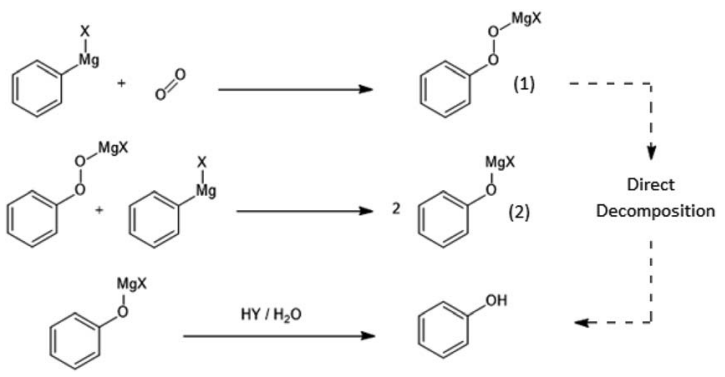

Fig. 5 Reaction of phenylmagnesium halide with oxygen to yield phenol. Note that while it is, in principle, possible that the magnesium halide phenyl hydroperoxide salt (1) could have been converted into free phenyl hydroperoxide by the acid quench, this free phenyl peroxide is not thought to be stable in isolation, due to having low $\mathrm{O}-\mathrm{O}$ bond energy, and could therefore also be directly broken down by the acid to yield phenol. ${ }^{20}$ As mentioned in the safety section in the Experimental in this paper, this may not be the case with potential peroxide products of other Grignard reagents and the possibility of the production of shock-sensitive and explosive alkyl hydroperoxide compounds must be considered.

phenylmagnesium halide to produce one molecule of product, so the yields and selectivity of the two products were compared directly on the basis of the different concentrations of phenol and benzoic acid in the product mixture. This was determined by quantitative proton NMR spectroscopy, using a known concentration of protic DMSO as an internal standard and calibration reference. An example aromatic spectrum is shown in Fig. 6, showing the high degree of peak separation at $400 \mathrm{MHz}$ between the two main compounds.

The full spectrum shows the reaction to typically be very clean, with only trace residual solvent peaks (acetone and tetrahydrofuran) in the aliphatic region of the spectrum. In many cases, only the phenol and benzoic acid peaks were visible in the aromatic region, aside from the residual solvent peak from the NMR solvent.

The peaks integrated for comparison between the two main products, which had the least noise throughout the experimental runs, were the ortho-protons, $\mathrm{H}_{\mathrm{A}}$ for both benzoic acid and phenol; 8.07-8.14 ppm and 6.85-6.89 ppm, respectively. While the proximity of the alcoholic proton to the ortho position in phenol may have been expected to slightly broaden the corresponding peak, altering the peak integration for measurement, the minor multiplicity of this peak was typically apparent, indicating that peak broadening would not be an issue for measurement in deuterated chloroform. Alternative comparisons between the meta and para peaks of both compounds were typically consistent with those of the ortho position, but as shown in Fig. 6, these were less accurate across the dataset due to the presence of the solvent peak and the lower intensity of the single proton para 




Fig. 6 Aromatic region of the NMR spectrum corresponding to the $5 \% \mathrm{O}_{2}, 13.6 \% \mathrm{CO}_{2}$ experiment, typical of most experiments.

position, particularly on the phenol and especially when the phenol production was low. The acidic proton for benzoic acid and the alcoholic phenol proton, not shown on Fig. 6, were identified as singlet peaks at $11.67 \mathrm{ppm}$ and $5.5 \mathrm{ppm}$ (broad), respectively, and are not suitable for comparison.

Of the trace by-products of the reaction, three main compounds were identified; a sharp peak at $7.36 \mathrm{ppm}$ which corresponded with traces of benzene produced from the hydrolysis of the Grignard reagent by trace water, which may have formed during work-up quench, during the course of the reaction or during storage of the Grignard (see Fig. 3). Note that this peak would be exaggerated on the proton NMR spectra by the 6-fold symmetry of the hydrogen atoms on the benzene molecule.

The only other major trace compounds identified in the reaction mixture were identified as benzophenone and triphenylmethanol. The former was identified via 2D COSY NMR, showing coupling between the multiplet at $7.82 \mathrm{ppm}$ and a complex multiplet at 7.47-7.57 ppm, which is obscured by the much stronger benzoic acid $\mathrm{H}_{\mathrm{B}}$ signal at the same position, and the latter triphenylmethanol was inferred by the presence of the benzophenone and the broad multiplet signal at $7.34 \mathrm{ppm}$.

The production of benzophenone from carboxylic acid salts using Grignard reagents has been observed recently where the Grignard intermediate product benzoate salt was then reacted with organolithium reagents to form a variety of benzophenones. ${ }^{21}$ While the previous report required the organolithium reagent to produce the benzophenone product, this process may occur with Grignards alone at the low yields observed in this study. Correspondingly this would involve the reaction of the magnesium benzoate salt with a second molecule of phenylmagnesium bromide followed by elimination of magnesium oxide. However, other recent computational studies have suggested that reactions of aromatic Grignard reagents may instead follow a radical pathway, which could also account for the benzophenone. ${ }^{22}$ Once the benzophenone product appeared, it would then itself be vulnerable to reaction with a third molecule of phenylmagnesium halide to produce, after quenching, the triphenylmethanol (Fig. 7).

While these other compounds consume multiple stoichiometric equivalents of the Grignard, it should be emphasised that they are not generated in large 

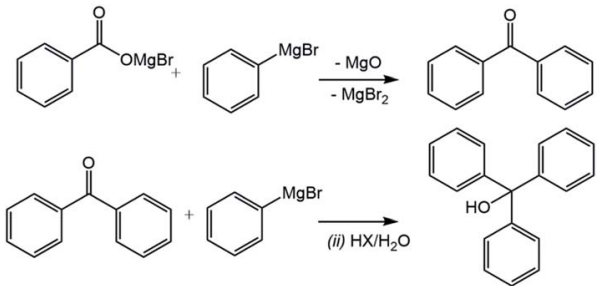

Fig. 7 Proposed pathway for the production of benzophenone and triphenylmethanol.

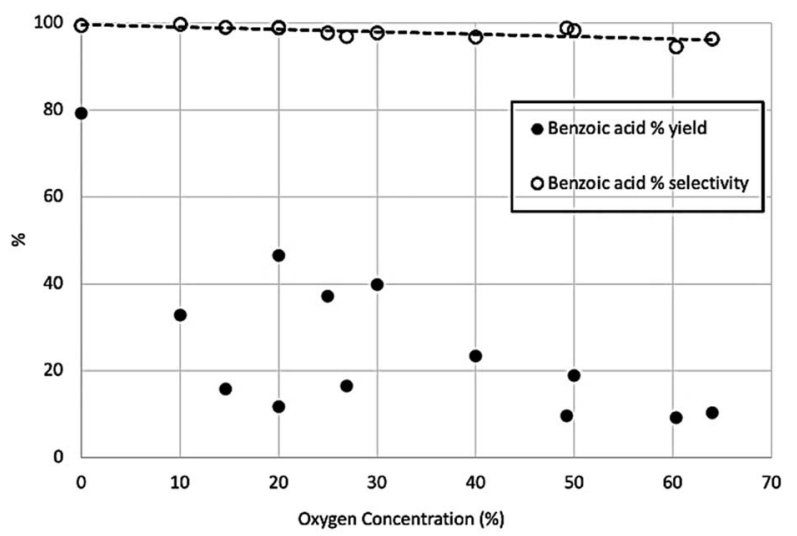

Fig. 8 Percentage of theoretical yield for benzoic acid production (solid circles) and percentage selectivity of benzoic acid over phenol production (open circles).

amounts, as discussed later. Across the range of $\mathrm{CO}_{2} / \mathrm{O}_{2}$ mixtures used, selectivity towards benzoic acid over phenol remained very high, above 95\% in all cases, although there was a gradual and marginal decrease in selectivity at high oxygen concentrations, while the yield of benzoic acid reduces significantly at higher oxygen concentrations (Fig. 8).

This shows the yield and selectivity for benzoic acid synthesis as a function of oxygen concentration in a mixture with carbon dioxide. While this may initially appear to show breakdown of the Grignard reagent there was no corresponding increase in phenol or any other aromatic compounds within the NMR trace. Previous studies have shown Grignard carbonation reactions to be first order with respect to carbon dioxide in the gas phase. Therefore, it is expected that as the oxygen concentration increases, and $\mathrm{CO}_{2}$ concentration decreases, the yield of benzoic acid per unit time will decrease, given the fixed volume of gas being used. Unfortunately, it was not possible to safely add further gas to the reaction mixture in this study, although we predict that with sufficient gas supply, the reaction would run to completion, even at the highest oxygen content levels. By plotting the natural $\log$ of the benzoic acid yield against $\mathrm{CO}_{2}$ concentration, a linear trend line can be fitted, passing through the $(0,0)$ point as shown in Fig. 9, which corresponds with the first order reaction profile. The small selectivity decrease with increasing oxygen concentration (Fig. 8) can therefore be seen as a consequence of decreasing benzoic acid yield caused by decreasing $\mathrm{CO}_{2}$ concentration 


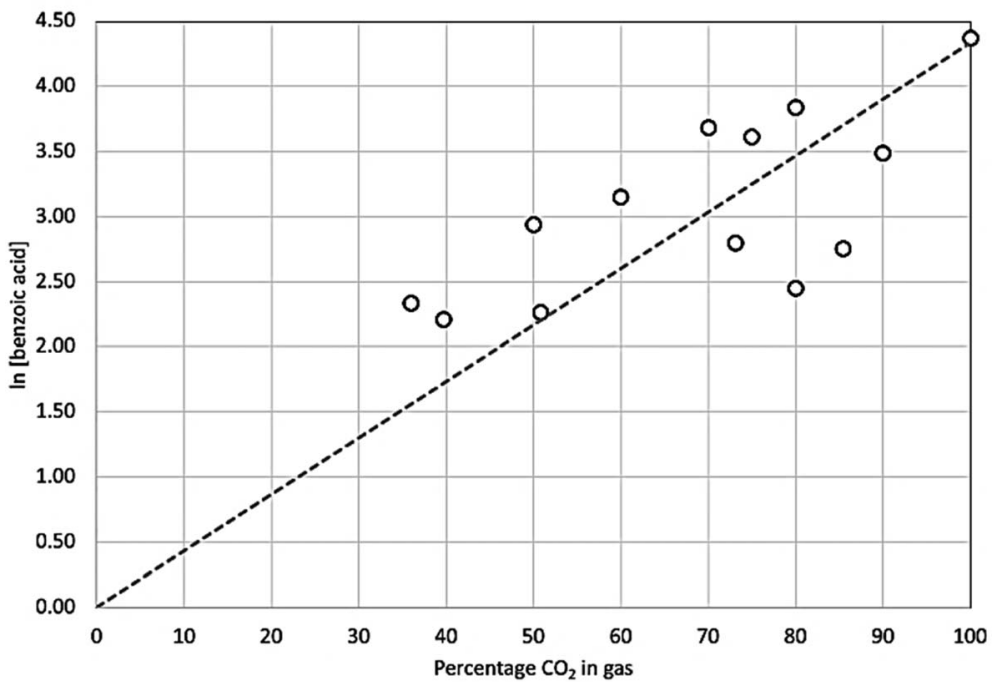

Fig. 9 Natural log of benzoic acid product concentration as a function of carbon dioxide concentration in the supply gas. Trend line projected back to point $(0,0)$.

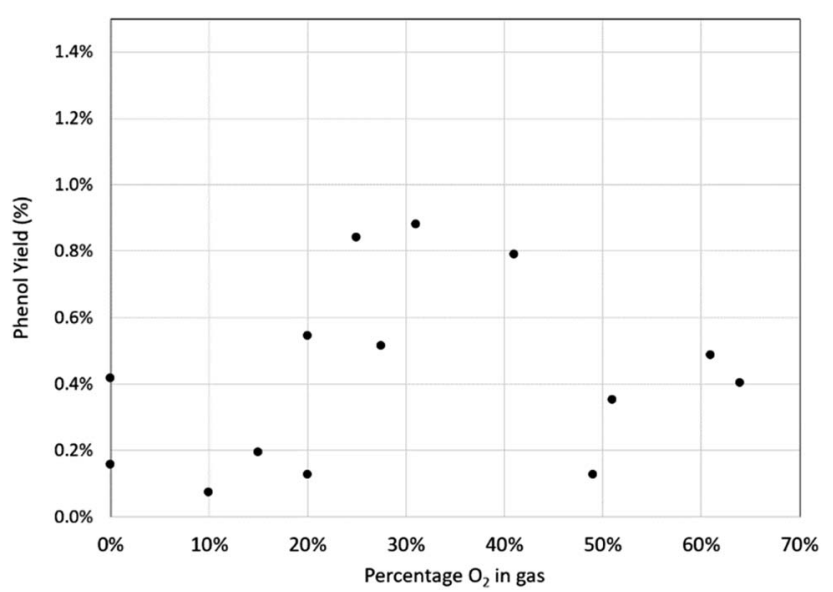

Fig. 10 Yield of phenol as a function of oxygen concentration.

in the feed gas, rather than a significant increase in phenol yield, which only varied between $0.1-0.9 \%$ across the range of conditions, with no clear trend with increasing oxygen concentration (Fig. 10).

Indeed, the two results for $0 \%$ oxygen concentration $\left(100 \% \mathrm{CO}_{2}\right)$ both show small quantities of phenol produced $(0.16 \%$ and $0.42 \%)$ and the greater of these two results exceeds the production of phenol at around $50 \%$ oxygen concentration $(0.12 \%$ and $0.35 \%)$, which calls into question whether atmospheric oxygen has any effect on the production of phenol at all and if perhaps a small amount of phenol is almost unavoidable.

Similarly, the yield of benzophenone appears to be largely independent of the oxygen concentration and is again present when there is no intentional oxygen 


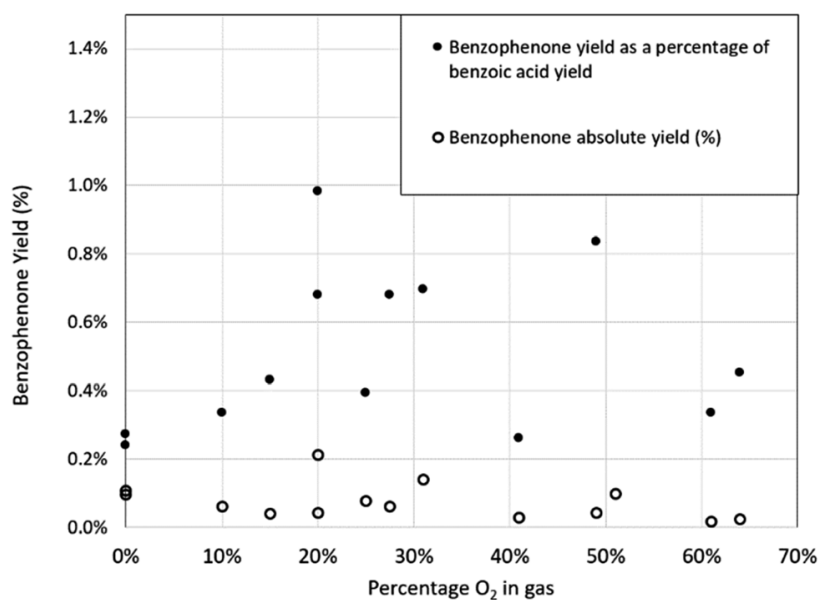

Fig. 11 Absolute yield of benzophenone and the yield of benzophenone as a percentage of benzoic acid yield as a function of oxygen concentration.

exposure. In Fig. 11, the absolute yield of benzophenone, as a percentage of the maximum possible yield of benzophenone, and the relative yield of benzophenone compared to the yield of benzoic acid is plotted. The weak trend of the absolute yield (hollow circles) reducing at elevated oxygen concentration gives similarly weak support to the proposed pathway of the benzoate intermediate of benzoic acid being the original source of the benzophenone, as shown in Fig. 7. However, the precise mechanism for the formation of benzophenone remains unclear, which is not uncommon in Grignard chemistry. ${ }^{22}$ The amount of suspected triphenylmethanol identified in the reaction mixture was not quantified due to peak overlap with other trace peaks including benzene, which made yield measurements by this method uncertain.

Overall, the results have consistently shown that even in the presence of large amounts of oxygen, the reaction of the Grignard with $\mathrm{CO}_{2}$ is dominant and oxygen poses no fundamental barrier to the desired reaction. This in turn, preliminarily indicates that otherwise clean (in terms of acidic gases) and dry flue gas could potentially be used in the reactive direct air capture of $\mathrm{CO}_{2}$ with phenyl Grignard, although low $\mathrm{CO}_{2}$ concentrations may suffer from slow reaction rates.

\section{Experimental}

\section{Safety notice}

The experiments detailed in this paper involve the deliberate exposure of pyrophoric Grignard reagents in highly flammable ethereal solution to oxygen, leading to an extremely high potential risk of fire if care is not taken. In most of the experiments, the concentration of oxygen did not exceed that of air $(21 \%$ volume) and the presence of carbon dioxide in concentrations above $35 \%$ will suppress flame propagation at $25{ }^{\circ} \mathrm{C}$ and one atmosphere even with extremely flammable diethyl ether solvent. ${ }^{23}$ Note also that hazardous or explosive peroxide products could also be generated with some Grignard reagents. 
However, in order to reduce flammability further, the reaction temperature was reduced, and the less flammable tetrahydrofuran-solvated Grignard reagents used, instead of diethyl ether. As an additional precaution, fire extinguishing media was always present and the experiments with high levels of oxygen were carried out at reduced volume and under constant supervision.

\section{Reagents and gas mixture preparation}

All Grignard reagents, additional solvents, molecular sieves and acid were purchased from Sigma Aldrich and used without further purification. Gas cylinders of $\mathrm{CO}_{2}, \mathrm{O}_{2}, 15 \% \mathrm{CO}_{2} / \mathrm{N}_{2}$ and compressed air $\left(21 \% \mathrm{O}_{2} / \mathrm{N} 2\right)$ were supplied by BOC-Linde.

The individual gas mixtures were created using a $500 \mathrm{~mL}$ Swagelok sample cylinder (part no. 316L-HDF4-500) with two needle valves for controlled pressurisation and overpressure bleeding. This was connected directly to one of the gas cylinder regulators and pressurised to a predetermined gauge pressure, corresponding to the partial pressure of the gas in the final mixture at the final intended sample cylinder pressure. The sample cylinder was then connected to the next gas regulator and pressurised further. Final gas composition was then checked using a $\mathrm{CO}_{2}$ Meter SprintIR $100 \% \mathrm{CO}_{2}$ sensor to measure $\mathrm{CO}_{2}$ content. This was calibrated in turn using a pair of Bronkhorst $100 \mathrm{~mL} \mathrm{~min}^{-1}$ mass flow controllers to create and measure known $\mathrm{CO}_{2} / \mathrm{N}_{2}$ and $\mathrm{CO}_{2} / \mathrm{O}_{2}$ mixtures.

\section{NMR}

Three types of NMR were used to analyse the products formed: 1D H-NMR, 2D HNMR and carbon-13 NMR, all carried out on a Bruker Avance $400 \mathrm{MHz}$ spectrometer. A sample of product was weighed and dissolved in $2 \mathrm{~mL} \mathrm{D}_{2} \mathrm{O}$ or $\mathrm{CDCl}_{3}$ (both $99.9 \% \mathrm{D})$. As an internal standard and calibration reference, $10 \mu \mathrm{L}(0.156$ mmol) of protic DMSO was added to determine the amounts of each product present in the sample by relative signal intensity, correcting by functional group proton number.

\section{Grignard reaction method}

A $100 \mathrm{~mL}$ 2-necked round-bottomed flask was thoroughly cleaned before being oven-dried at $70-80{ }^{\circ} \mathrm{C}$ for 16 hours. A magnetic stirrer bar was placed in the flask, and the vertical neck connected, via a condenser, to a nitrogen Schlenk line with a silicone oil exhaust bubbler. The side arm of the flask was stoppered. Using standard Schlenk line techniques, the line, condenser and reaction flask were subjected to three cycles of nitrogen purging and vacuum pumping. Under a strong flow of nitrogen, a pierce-able stopper was placed into the side arm. Through this stopper, under positive nitrogen pressure, $10 \mathrm{mmol}$ of $2 \mathrm{M}$ phenylmagnesium chloride or $1 \mathrm{M}$ phenylmagnesium bromide, both in THF solution, were added via syringe through the seal. For binary $\left(\mathrm{CO}_{2} / \mathrm{O}_{2}\right)$ gas mixtures, $10 \mathrm{mmol}$ of Grignard reagent was used; for ternary systems, where the $\mathrm{CO}_{2} / \mathrm{O}_{2}$ ratio was lower, this was reduced for safety to $5 \mathrm{mmol}$. HPLC grade 2-MeTHF, dried over $3 \AA$ molecular sieves, was added to the flask in a volume ratio of $3: 1$ with respect to phenylmagnesium halide. This was added to prevent fouling of the magnetic follower from the increase in viscosity of the reaction mixture during the reaction. Despite the highly exothermic reaction, the temperature of the 




Fig. 12 Diagram of Grignard reaction apparatus. Note that the additional ports on the Schlenk line cannot be used during or immediately after this type of experiment.

reaction was maintained below $25^{\circ} \mathrm{C}$ by immersing the flask in a cold-water bath. The nitrogen supply from the Schlenk line was then stopped and the gas mixture was slowly sparged through the reaction mixture, via a long needle positioned close to the magnetic follower, with the gas bubbler attached to the Schlenk line. Note that this process contaminates the non-vacuum side of the Schlenk line with the reaction off-gases and any other ports on the line should be closed and not used for other work until the line has been thoroughly purged with the usual inert gas (Fig. 12).

As the Grignard will react rapidly with the $\mathrm{CO}_{2}$ atmosphere being introduced, the initial flow rate must be high enough to prevent air from being drawn back through the bubbler as a negative atmospheric gauge pressure can be easily achieved. However, this process has allowed for monitoring the reaction progress in cases where there was an abundant supply of $\mathrm{CO}_{2}$ gas. This monitoring was achieved by briefly interrupting the gas supply and observing the pressure drop within the experimental apparatus. Exhaustion of the Grignard reagent was previously typically indicated by the pressure within the reaction vessel failing to decrease, as indicated by the position of the oil level in the exhaust bubbler although additional time must be given to account for thermal gas expansion in the event the gas flow rate has caused the reaction mixture to become overly cold. ${ }^{22}$ However, at lower $\mathrm{CO}_{2}$ concentrations, however, this approach was not found to be reliable, as the reduced reaction rate at low $\mathrm{CO}_{2}$ concentration was preventing the Grignard reagent from being consumed before the gas bottle was exhausted, for safety reasons, it was not possible to refill the gas bottle for continuation of the reaction in this study. The initial high flow rate was reduced as the reaction progressed and reaction rate diminished, to prevent excessive 
Grignard solvent evaporation. It is however also worth noting that the solvent evaporation through the sparging process provided further evaporative cooling to the reaction mixture.

After the reaction, $4 \mathrm{M}$ aqueous $\mathrm{HCl}$ was carefully added until the reaction was quenched and the precipitate of magnesium bromobenzoate was converted into soluble benzoic acid and magnesium bromochloride. This acid addition will also break down any peroxides produced from side-reactions. The mixture was then evaporated to dryness under vacuum by rotary evaporation under mild heating (less than $40{ }^{\circ} \mathrm{C}$ ) to prevent evaporation of any phenol generated during the reaction, then the mixture was filtered from acetone to remove the magnesium salts before being again dried overnight at room temperature to give a white microcrystalline powder.

\section{Conclusions}

Building on a previous study into the possibility of using Grignard agents for direct capture and conversion of $\mathrm{CO}_{2}$, the effect of oxygen in the gas stream on the reactivity of dilute $\mathrm{CO}_{2}$ has been investigated. This would, in principle, allow the simplification of the capture and conversion process, as well as allow the carbon dioxide utilisation (CDU) to occur at room temperature and atmospheric pressures, by essentially allowing a looped magnesium cycle to drive the process. The magnesium dihalide by-product of the reaction could then be directly recycled using the well-developed industrial route for magnesium metal production. Through the value-addition of the $\mathrm{CO}_{2}$ molecule into an existing organic structure, facile routes to profitable CDU chemicals may be developed, potentially paving the way for bulk CDU products to be brought to market that may use a climate-relevant quantity of emissions in their manufacture.

By carefully conducting a series of experiments on the reaction of Grignard reagents with industrially relevant concentrations of $\mathrm{CO}_{2} / \mathrm{O}_{2}$ gas mixtures, a comparison between the relative reactivity of the two gases was made. This demonstrated that phenyl Grignard will preferentially react with carbon dioxide to a very high selectivity, even when the oxygen is at significantly higher concentrations than $\mathrm{CO}_{2}$. Indeed, the concentration of oxygen appears to have little measurable effect on the rate of the oxygen-based reaction of phenyl Grignard to phenol. The largest effect of high-oxygen content gases appeared to be their simple dilution of the $\mathrm{CO}_{2}$ stream, only reducing the overall rate of the Grignard reaction.

Benzophenone and triphenylmethanol were also detected in the reaction mixture at low concentrations. The production of benzophenone, similarly to phenol, appeared to be independent of the oxygen concentration but may have some weak dependence on the concentration of the intermediate magnesium benzoate bromide salt. This provides some weak evidence of a double reaction of phenylmagnesium bromide with $\mathrm{CO}_{2}$, followed by the tripling of phenyl groups to produce triphenylmethanol after acidic workup. This would be consistent with the recent report of benzophenone production from the Grignard reaction with carbonate salts followed by secondary reaction with organolithium, but at much lower yield to benzophenone. However, the mechanism of this process, as with much of Grignard chemistry, is not fully settled. 


\section{Author contributions}

George Dowson: supervision, laboratory work, NMR, co-writing of the original draft; Joshua Cooper: laboratory work as MEng research student; Peter Styring: conceptualisation, kinetics, supervision, co-writing of the original draft.

\section{Conflicts of interest}

The authors declare no conflicts of interest.

\section{Acknowledgements}

We wish to thank UKRI-EPSRC for funding which enabled the execution of the research. In particular, we acknowledge funding from the 4CU Programme Grant (EP/K001329/1), CO2Chem Network (EP/P026435/1) and the SUSTAIN Manufacturing Hub (EP/S018107/1).

\section{Notes and references}

1 P. Styring, Carbon dioxide utilization as a mitigation tool, in Managing Global Warming, Academic Press, Cambridge, MA, 2019.

2 A. Zimmermann, L. J. Müller, A. Marxen, K. Armstrong, G. Buchner, J. Wunderlich, A. Kätelhön, M. Bachmann, A. Sternberg, S. Michailos, H. Naims, P. Styring, R. Schomäcker, A. Bardow and V. Sick, TechnoEconomic Assessment \& Life-Cycle Assessment Guidelines for CO2 Utilization, CO2Chem Media \& Publishing Limited, Sheffield, UK, 2018.

3 S. R. Haszeldine, Science, 2009, 325, 1647-1652.

4 K. Li, W. Leigh, P. Feron, H. Yu and M. Tade, Appl. Energy, 2016, 165, 648-659.

5 MEA capture energy data taken from: T. Harkin, A. Hoadley and B. Hooper, Energy Procedia, 2009, 1, 3817-3825; L. M. Romeo, I. Bolea and J. M. Escosa, Appl. Therm. Eng., 2008, 28, 1039-1046; Y. Yang and R. Zhai, Paths to Sustainable Energy, ed. J. Nathwani and A. Ng, InTech, Rijeka, Croatia, 2010, ch. 24, pp. 499-510; U. Desideri and A. Paolucci, Energy Convers. Manage., 1999, 40, 1899-1915.

6 Figures calculated from the standard Gibbs energy of reaction using NIST Webbook figures for standard enthalpy and entropy; M. W. Chase, Thermochemical Data, in NIST Chemistry Webbook, NIST Standard Reference Database Number 69, ed. P. J. Linstrom and W. G. Mallard, National Insititute of Standards and Technology, Gaithersburg, Maryland, USA, retrieved $3^{\text {rd }}$ Janary 2021.

7 P. Styring, Carbon Dioxide Utilisation: Volume 1 Fundamentals, ed. M. North and P. Styring, de Gruyter, Berlin, 2019, p. 204.

8 G. R. M. Dowson, I. Dimitriou, R. E. Owen, D. G. Reed, R. W. K. Allen and P. Styring, Faraday Discuss., 2015, 183, 47-65.

9 G. Demirci and I. Karakaya, Magnesium Technology, ed. S. N. Mathaudhu, W. H. Sillekens, N. R. Neelameggham and N. Hort, John Wiley \& Sons, Hoboken, NJ, USA, 2012, pp. 59-62.

10 Calculated from the energy cost of the production of $0.552 \mathrm{~kg}$ magnesium using figures from; I. J. Polmear, Magnesium and Magnesium Alloys, 
ed. M. M. Avedesian and H. Baker, ASM International, Ohio, USA, 1999 and G. Demirci and I. Karakaya, Magnesium technology, ed. S. N. Mathaudhu, W. H. Sillekens, N. R. Nellameggham and N. Hort, John Wiley \& Sons, Hoboken, New Jersey, USA, 2012, pp. 59-62.

11 Th. M. Wortel, D. Oudijn, C. J. Vleugel, D. P. Roelfosen and H. van Bekkum, J. Catal., 1979, 60, 100-120.

12 V. Adamian and W. H. Gong, Chemistry and Mechanism of Oxidation of paraXylene to Terephthalic Acid using Co-Mn-Br Catalyst, in Liquid Phase Aerobic Oxidation Catalysis, ed. S. S. Stahl and P. L. Alders, Wiley VCH, 2016, pp. 41-66.

13 Average FOB Benzene price for 2017 of US\$822-US\$860 per tonne. Argus Benzene Annual 2018 Introduction, Argus Media, London, UK, 2018. Average US Terephthalic acid trading price for 2017 of $\$ 920$ per ton (US\$1,015 per tonne), Global purified Terephthalic Acid (PTA) Market Insight, Plastics Insight, Noida, India, 2019.

14 A. Buchard, M. North, C. Kozak, E. Remiezowicz, P. Abrantes, A. Bardow, J. Dodson, C. Williams, J. Albo, I. Ingram, G. V. S. M. Carrera, P. Styring, M. Poliakoff, K. Lamb, E. A. Quadrelli, N. MacDowell, G. Dowson, M. Aresta, R. Heyn, J. A. Castro-Osma, W. Webb, R. Silva, A. Marciniak, C. Jones, C. T. Yavuz, C. Capacchione, A. Coogan and M. Priestnall, Faraday Discuss., 2015, 183, 97-123.

15 A. Heyn, Fire protection concept for the storage of pyrophoric materials, IChemE Symposium Series 160, Hazards 25, Edinburgh International Conference Centre, Edinburgh, UK, Bayer, 2015, vol. 77, pp. 6032-6038.

16 C. Walling and S. A. Buckler, J. Am. Chem. Soc., 1955, 22, 6039-6041.

17 A. J. Kidnay, W. R. Parrish and D. G. McCartney, Fundamentals of Natural Gas Processing, CRC Press, Boca Raton, Florida, USA, 2020, pp. 319-320.

18 Dry values for each industry were averaged from each source. When wet values $(x)$ are reported, the calculated dry value $(y)$ is calculated as follows: $y=100 x$ / $100-(\%$ fraction water). Coal, values for lignite, sub-bituminous and black: Y. Tan, E. Croiset, M. A. Douglas and K. V. Thambimuthu, Fuel, 2006, 85(4), 507-512; K. C. Galbreath and C. J. Zygarlicke, Fuel Process. Technol., 2000, 65-66, 289-310; CSIRO Advanced Coal, Environmental Impacts of Amine-based $\mathrm{CO}_{2}$ Post Combustion Capture (PCC) Process, CSIRO, Canberra, Australia, 2012; E. P. Robertson, Analysis of CO2 Separation from Flue Gas, Pipeline Transportation, and Sequestration in Coal, USDOE, INL, Idaho, USA, 2007; R. Zevenhoven and P. Kilpinen, Control of pollutans in flue gases and fuel gases, Helsinki Finland, 2001Coal and oil power flue gas figure: M. R. Beychok, Fundamentals of stack gas dispersion, M. Beychok, California, USA, 2005Gas power figure: Zevenhoven \& Kilpinen, 2001; Beijing Jiaotong University and North China Electric Power University, People's Republic of China: Study on Carbon Capture and Storage in Natural Gas-Based Power Plants, Beijing, China, 2014Waste incineration gas composition figure: Zevenhoven \& Kilpinen, 2001; U. Jecht and R. Bürssner, Flue Gas Analysis in Industry, Practical guide for Emission and Process Measurements, Testo, Lenzkirch, Germany, 2012, pp. 82-84; Cement plant figure: Zevenhoven \& Kilpinen, 2001Steel plant figures (various processes): C.-Y. Kao, T.-Y. Chen, Y.-B. Chang, T.-W. Chiu, H.-Y. Lin, C.-D. Chen, J.-S. Chang and C.-S. Lin, Bioresour. Technol., 2014, 166, 485-493; A. Tobiesen, K. A. Hoff, G. Haugen, 
T. Mejdell, Process Evaluations and Simulations of $\mathrm{CO}_{2}$ Capture from Steel Plant Flue Gases, Industry CCS Workshop, Düsseldorf, Germany, 2011.

19 Z. He and T. F. Jamison, Angew. Chem., Int. Ed., 2014, 53, 3353-3357.

20 N. Sebbar, H. Bockhorh and J. W. Bozzelli, Phys. Chem. Chem. Phys., 2002, 4, 3691-3703.

21 T. E. Hurst, J. A. Deichert, L. Kapeniak, R. Lee, J. Harris, P. G. Jessop and V. Snieckus, Org. Lett., 2019, 21, 3882-3885.

22 R. M. Peltzer, J. Gauss, O. Eisenstein and M. Cascella, J. Am. Chem. Soc., 2020, 142, 2984-2994.

23 M. G. Zabetakis, Flammability characteristics of combustible gases, 627, U.S. Department of the Interior, Bureau of Mines, Washington D.C., USA, 1964. 